\title{
First Real-World Insights into Belimumab Use and Outcomes in Routine Clinical Care of Systemic Lupus Erythematosus in Germany: Results from the OBSErve Germany Study
}

\author{
Andreas Schwarting • Johann O. Schroeder • Tobias Alexander • Marc Schmalzing • \\ Christoph Fiehn · Christof Specker · Alessandra Perna · Constanze Cholmakow-Bodechtel • \\ Volker B. Koscielny · Heike Carnarius
}

Received: April 15, 2016/Published online: November 1, 2016

(C) The Author(s) 2016. This article is published with open access at Springerlink.com

\begin{abstract}
OBSErve Germany was the first observational study of belimumab as add-on treatment for systemic lupus erythematosus (SLE) in routine clinical care in Germany, retrospectively collecting data from 102 SLE patients, 6 months before and after belimumab initiation. Most patients had moderate or
\end{abstract}

Enhanced content To view enhanced content for this article go to http://www.medengine.com/Redeem/ 7017F06060C5FE6E.

A. Schwarting $(\bowtie)$

Department of Rheumatology and Clinical Immunology, University Medical Centre of the Johannes Gutenberg-University Mainz, ACURA Center for Rheumatic Diseases Bad Kreuznach, Mainz, Germany

e-mail: schwarting@uni-mainz.de

J. O. Schroeder

Department of Internal Medicine 1, University

Hospital Schleswig-Holstein, Campus Kiel, Kiel,

Germany

T. Alexander

Department of Rheumatology and Clinical

Immunology, Charité-University Medicine Berlin,

Berlin, Germany

M. Schmalzing

Department of Internal Medicine 2, University

Hospital, Würzburg, Germany severe SLE and several SLE manifestations. After 6 months of belimumab treatment, 78\% of patients showed an improvement in overall disease activity of at least $20 \%$ in their physician's judgment and for $42 \%$ of patients the improvement was at least 50\%. Similar results were observed for the most common manifestations: arthritis, fatigue, rash, alopecia, increased anti-dsDNA antibody levels, and low complement. The SLE Disease Activity Index (SLEDAI/SELENA-SLEDAI) decreased from 10.6 to $5.6(n=65)$, with other indices also showing

C. Fiehn

ACURA Center for Rheumatic Diseases,

Baden-Baden, Germany

C. Specker

Department of Rheumatology and Clinical Immunology, University Hospital, Essen, Germany

A. Perna · V. B. Koscielny

GF Immuno Inflammation/ID Franchise,

GlaxoSmithKline, Brentford, UK

C. Cholmakow-Bodechtel

Public Health, Kantar Health, Munich, Germany

H. Carnarius

Medical Department, Immunology and Rare

Diseases Business Unit, GlaxoSmithKline, Hamburg, Germany 
improvement. A notable dose reduction was seen for concomitant oral corticosteroids, from 13.7 to $7.6 \mathrm{mg} /$ day overall $(n=91)$, and from 17.5 to $8.6 \mathrm{mg} /$ day in patients with a high corticosteroid dose at belimumab initiation $(\geq 7.5 \mathrm{mg} ; n=63)$. Six patients discontinued belimumab therapy within 6 months. Overall, belimumab showed promising results for SLE patients in real-world settings. After 6 months of belimumab treatment, disease activity and corticosteroid use were reduced. The discontinuation rate was low and belimumab appeared to be well tolerated.

Funding GlaxoSmithKline UK.

Keywords: B cell targeted therapy; Belimumab; Observational study; SLE; Systemic lupus erythematosus

\section{INTRODUCTION}

Systemic lupus erythematosus (SLE) is an autoimmune disease with heterogeneous morbidities, involving the skin, joints, kidneys, or central nervous system. The clinical course is characterized by chronic remitting and relapsing phases which usually requires life-long medication to manage disease activity. Standard medications for SLE include oral corticosteroids, antimalarials, cytotoxic agents, non-steroidal anti-inflammatory drugs (NSAIDs), and immunomodulators/immunosuppressors, some of which can lead to severe side effects, especially at higher doses [1-7]. Potentially severe complications of SLE include lupus nephritis and central nervous system vasculitis, both of which require long-term treatment with high doses of corticosteroids and/or cyclophosphamide and tend to relapse $[1,8]$. The combination of SLE manifestations and side effects of long-term medication use can severely impact on the patients' quality of life and ability to work. However, advances in therapy and management have increased life expectancy and quality of life for SLE patients $[9,10]$.

New types of therapies include biologicals $[11$, 12], e.g., the BLyS (B lymphocyte stimulator) inhibitor belimumab (trade name Benlysta $\left.^{\circledR}\right)$, a human monoclonal $\operatorname{IgG} 1 \lambda$ antibody that is indicated for adult autoantibody-positive SLE patients that require an add-on to other SLE medications due to a persisting high degree of disease activity despite standard therapy, e.g., in cases with ineffectiveness or intolerability of other medications [13]. Belimumab received market authorization for Europe and the USA in 2011, after safety and efficacy were confirmed in two randomized, placebo-controlled clinical trials [14-18], subsequently followed by an ongoing continuation study [19]. At this stage, it is essential to evaluate how the results of these studies translate into the clinical reality of managing and treating SLE patients.

BLyS is an essential B cell survival factor acting by binding to specific B cell surface receptors. In SLE, an excess concentration of BLyS inhibits the physiological apoptosis of autoreactive B cells and promotes their pathological differentiation into autoantibody-producing plasma cells. Belimumab acts by capturing and inhibiting soluble BLyS before it binds to its receptors, thus indirectly inhibiting the survival and differentiation of autoreactive B cells, as well as the subsequent production of autoantibodies by plasma cells [20, 21]. Belimumab is a long-term treatment administered as an intravenous infusion, with the initial three doses given every 2 weeks (week 0, 2, 4) followed by 4-weekly administrations. Clinical response has been described as early as 8 weeks [22]. However, generally patients should be treated for 
6 months before a decision about treatment discontinuation due to lack of response is taken [13]. Patients with high disease activity levels despite standard therapy have been shown to benefit most from belimumab treatment, especially regarding clinical disease activity (e.g., as measured by validated scores), corticosteroid dependence, and laboratory markers such as autoantibody-positivity or low complement levels [16].

OBSErve is a multinational study programme with results having been reported from the USA and Spain so far [23, 24]. The present study, OBSErve Germany (Evaluation Of Use of Belimumab in Clinical Practice Settings; GSK study number GSK 117214), is part of this programme and the first real-world observation of belimumab use and outcomes in routine clinical care in Germany, 2 years after belimumab was launched on the European market. Further OBSErve studies are under way or planned in other European and Latin American countries. The objective of the study was to observe the overall patterns of SLE care and outcomes among belimumab users in clinical practice in Germany and to describe the belimumab patient population, the reasons for initiating and discontinuing belimumab treatment, aspects of healthcare resource utilization in these patients, and the use of routine disease assessment tools in clinical practice. After the clinical trials confirmed efficacy and safety in an interventional setting, it is important to investigate usage patterns and disease management results for patients in routine care, as assessed by their treating physicians [25]. For this study, treatment results were analyzed after the initial 6 months of belimumab therapy, in line with the summary of product characteristics for belimumab which recommends a minimum treatment period of
6 months before a decision about the treatment outcomes and its continuation can be made [13].

\section{METHODS}

\section{Study Design}

OBSErve Germany was a retrospective, multicenter, observational cohort study collecting data on belimumab therapy in routine care of SLE patients in Germany. It was designed to gather real-world information from patient medical records on the short-term (6 months) clinical outcome in SLE patients treated with belimumab. The study was conducted in line with the Guidelines for Good Pharmacoepidemiology Practices (GPP). All study documents were submitted to the responsible ethics committee (EC) of the local coordinating investigator, i.e., the Landesaerztekammer Rheinland-Pfalz. According to the local requirements, the observational plan was notified to the competent higher federal authority, the Paul Ehrlich Institute (PEI) before starting data collection. Data collection for the study was performed between April 2013 and November 2013 in 21 rheumatology practices. During the data collection period, monthly update reports on the participating physicians were provided to the Federal Association of Panel Doctors (KBV), the Central Federal Association of the Health Insurance Funds (GKV-Spitzenverband), and the Association of Private Health Insurance Funds (PKV) according to national law AMG $\S 67(6)$. As a result of its retrospective character, this study did not influence the physicians' treatment decisions; frequency and timing of examinations and the prescription of belimumab were handled according to the 
physician's routine practice and no additional examinations were required for the study. Therefore patient's informed consent was not required.

\section{Study Population}

OBSErve Germany was sponsored by GSK who contributed their knowledge about sites with experience in prescribing belimumab to be contacted with a feasibility questionnaire. According to the predefined eligibility criteria, the study sample included clinical sites which had at least 5 years of experience in treating SLE patients and at least 6 months experience in the usage of belimumab for SLE. At the start of the study the participating physicians were required to be managing at least ten SLE patients at the time and to have initiated belimumab in at least two patients as part of routine care, with at least one of them still receiving belimumab at the time.

Patients included in the documentation had to be at least 18 years old and belimumab-naïve with a confirmed diagnosis of SLE. For these patients, treatment with belimumab had to be initiated as part of routine care at least 6 months before inclusion into the study, ensuring a purely retrospective observation and documentation of the first 6 months of the treatment. All eligible patients had to be included at each site, also if they discontinued belimumab therapy within the initial 6 months, in order to also obtain insight into the frequency and reasons for early discontinuation in a real-world setting. Data that had to be available included medical and treatment history for at least 6 months prior to belimumab initiation, the reason for belimumab initiation, treatment outcomes at 6 months after start of treatment, and the reason for discontinuation if belimumab was discontinued. Exclusion criteria were current enrollment in an SLE-related trial or initiation of belimumab therapy as part of a clinical trial interventional arm.

\section{Study Conduct}

For this study, the index date was defined as the date of belimumab initiation. The study period included a treatment history period of 6 months prior to the index date followed by the initial belimumab treatment period of 6 months after the index date. This allowed for an observation time of about 12 months for each patient in the study. The participating physicians identified all adult SLE patients in their practices who fulfilled the eligibility criteria, to avoid selection bias. The physicians collected and documented the relevant information from their patients' medical records in anonymized form. Information on the physicians' practices and their SLE management approaches were collected, including practice type, patient caseload, and routine SLE diagnosis and management approaches. The following patient data from medical records were captured in the patient case report form (CRF): patient demographics, co-morbidities, SLE disease characteristics at start of belimumab therapy, belimumab therapy, other SLE and non-SLE medications, outcomes related to clinical and serological manifestations, disease activity assessments, physician judgment on overall clinical response 6 months after initiation, and healthcare resource utilization.

All assessment results regarding disease activity and outcome (e.g., the "Safety of Estrogens in Lupus Erythematosus National Assessment" (SELENA) modification of the SLE Disease Activity Index (SLEDAI)) were estimated by the participating physicians themselves, either immediately during a patient's visit, or 
retrospectively from the patient chart. At the time of initiating belimumab the scoring used ranged from mild, moderate to severe lupus. Regarding the treatment effects 6 months after belimumab the physicians' judgment scale for disease activity, overall as well as based on disease manifestations ranged from worse, no improvement, less than $20 \%$ improvement (i.e., minimally improved), 20-49\% improvement (i.e., clear but moderate improvement), 50-79\% improvement (i.e., great improvement) to at least $80 \%$ improvement (i.e., nearly normalized). This estimated score was also the primary endpoint measure to reflect the physicians' opinion of the improvement of their patients' SLE status. This scale was not a validated instrument but aimed to reflect the real-world clinical approach for the evaluation of treatment effectiveness, where formal instruments to assess disease activity (such as the SLEDAI or ECLAM (European Consensus Lupus Activity Measurement Index)) are not yet broadly used on a regular basis, despite recommendations by the European League Against Rheumatism (EULAR) [26]. Therefore, the physicians' judgment scale, used identically across the whole OBSErve study programme, aimed to obtain a subjective clinical rating.

Additionally, disease assessments using Physician Global Assessment Scales and Patient Global Assessment Scales were collected where available. The physicians who documented results from these scales used their internal rating system and the results were then converted to a scale ranging from 0 to 100 , based on the ranges provided by the physicians.

Data collection was performed using an electronic data capture system (EDC). Automatic checks were implemented in the EDC to avoid missing answers and to provide valid and plausible data entries. During the study it was discovered that data related to concomitant SLE medication were incomplete. Thus, queries were sent out to the respective physicians to confirm and to complete the provided information.

\section{Data Analysis}

All statistical analyses were performed in a descriptive manner and were interpreted in an explorative way. Tables were created with the software SAS $^{\circledR}$ version 9.2 for Windows. For continuous data, the number of non-missing and missing values, mean, standard deviation, minimum, median, and maximum were calculated. For the analysis of corticosteroid doses and for SLEDAI/SELENA-SLEDAI response groups, values of continuous variables were additionally presented as categorical data. The analysis included all valid cases (fulfilling the inclusion criteria, CRFs completed) from eligible sites (fulfilling the inclusion criteria). The evaluation of treatment outcome data after 6 months was performed for all patients who completed the initial 6 months of belimumab therapy, in line with the summary of product characteristics for belimumab which recommends a minimum initial treatment period of 6 months [13].

\section{Compliance with Ethics Guidelines}

This article does not contain any new studies with human or animal subjects performed by any of the authors.

\section{RESULTS}

\section{Baseline Characteristics of Study Sites}

For this study, SLE patient data and treatment outcomes under belimumab therapy were 
collected retrospectively for 102 patients from 21 sites in Germany, mainly hospitals (76\%). On average (mean \pm standard deviation), the physicians' experience in treating SLE patients was $15.7 \pm 6.74$ years ( $\min 5, \max 30$ years). They were treating $72.7 \pm 30.51$ SLE patients (min 20, max 100 patients) at the time of data collection. Each physician, on average, was treating $5.7 \pm 4.73$ patients with belimumab. Of these, $4.8 \pm 2.17$ patients had been receiving belimumab for at least 6 months.

The most common disease assessment tools used routinely by the study sites for SLE management were the SLEDAI (57\%)/ SELENA-SLEDAI (29\%), the Patient Global Assessment Scale (43\%), the ECLAM (38\%), and the Physician Global Assessment Scale (33\%). Furthermore, the most frequent routine laboratory analyses included testing of C3 and C4 concentrations (performed by $100 \%$ of physicians), serum creatinine levels (100\%), C-reactive protein levels (95\%), as well as a complete blood count (95\%). The majority of participating physicians (62\%) stated that the patient's opinion was highly important when considering changes in the individual SLE therapy.

\section{Patient Baseline Characteristics and Reasons for Initiating Belimumab Therapy}

A total of 102 patients were included in the analysis. Most of them were female (91\%) and all but one were Caucasian. The mean age of the patients was $42.5 \pm 13.83$ years (min 19, max 76) and the mean body mass index was $25.3 \pm 6.19 \mathrm{~kg} / \mathrm{m}^{2}$. The SLE-related baseline data and the co-morbidities of the patients are provided in Table 1.

The patients' SLE disease severity before initiating belimumab treatment, i.e., at baseline, was assessed by their physician. The majority of patients had moderate $(60 \%)$ or severe (25\%) SLE and most (58\%) had been diagnosed with SLE more than 10 years ago. The most common laboratory findings for these patients at baseline were high levels of anti-dsDNA antibodies (in $72 \%$ of patients) and below-normal levels of the complement components $3(61 \%)$ and 4 (52\%).

The number of clinical and serological manifestations of SLE varied in the study population, but for $60 \%$ of patients, four or more manifestations were documented at baseline. The most frequently documented SLE manifestations at baseline were arthritis (67\% of patients), increased anti-dsDNA antibody levels (56\%), low complement levels (47\%), rash (40\%), lupus nephritis (25\%), and alopecia (25\%). The most frequently listed co-morbid conditions of the patients at the start of belimumab therapy were fatigue (41\%), hypertension (35\%), osteoporosis (20\%), and depression (12\%).

The indication for belimumab was related to mucocutaneous, arthritis, serositis, and mild lupus nephritis occurring with other lupus manifestations. The most common reason for initiating belimumab therapy was ineffectiveness of the patient's previous treatment regimen (88\% of patients). Further common reasons were a worsening of the patient's condition (61\%) and a desire to decrease the use of corticosteroid drugs ("steroid sparing") (40\%).

\section{SLE Disease Activity at Baseline}

The following comparisons of results from baseline to 6 months later are presented for all 96 patients who completed the initial 6 months of treatment. This reflects the recommendation made by the European regulatory authority (the 
Table 1 SLE disease baseline data and co-morbidities at time of start of belimumab therapy

Number of patients

$(N=102)$

SLE severity at belimumab therapy start ${ }^{\mathrm{a}}$

Mild

Moderate

Unknown

Time since SLE diagnosis (years)

$<1$

$1-5$

$6-10$

$>10$

Laboratory values at start of belimumab therapy ${ }^{\mathrm{b}}$

High anti-dsDNA antibody titres ${ }^{c}$

Thrombocytopenia

Hemolytic anaemia

None of these

Number of SLE manifestations at start of belimumab therapy

0

1

2

3

4

5

$>5$

SLE manifestations at start of belimumab therapy (most frequent $)^{\mathrm{b}}$

Arthritis

Increased anti-dsDNA antibody levels ${ }^{\mathrm{c}}$
Percentage of patients

\section{8}

60

25

7

1

19

58

72

61

52

29

17

15

6

9

99


Table 1 continued

\begin{tabular}{|c|c|c|}
\hline & $\begin{array}{l}\text { Number of patients } \\
(N=102)\end{array}$ & $\begin{array}{l}\text { Percentage of patients } \\
\text { (\%) }\end{array}$ \\
\hline Lupus nephritis & 26 & 25 \\
\hline Alopecia & 25 & 25 \\
\hline \multicolumn{3}{|l|}{ Co-morbidities (most frequent $)^{\mathrm{b}}$} \\
\hline Fatigue & 42 & 41 \\
\hline Hypertension & 36 & 35 \\
\hline Osteoporosis & 20 & 20 \\
\hline Depression & 12 & 12 \\
\hline None & 17 & 17 \\
\hline \multicolumn{3}{|l|}{ Reasons for start of belimumab therapy ${ }^{b}$} \\
\hline Previous treatment regimen not effective & 90 & 88 \\
\hline Patient condition worsening & 62 & 61 \\
\hline Decrease use of corticosteroids (steroid sparing) & 41 & 40 \\
\hline Previous treatment regimen not well tolerated & 27 & 26 \\
\hline Previous treatment regimen inconvenient & 3 & 3 \\
\hline Myositis & 1 & 1 \\
\hline
\end{tabular}

CH50 total complement activity assay, CRF case report form, $d s D N A$ double-stranded DNA, SLE systemic lupus erythematosus

a Subjective retrospective categorization of patient's status at baseline by physician

b Multiple responses possible

c Discrepancies in the incidence of high antibody titres and low complement levels between the categories "laboratory values" and "SLE manifestations" in this table are due to the fact that not all physicians may have considered these laboratory values as "manifestations". Furthermore, the physicians were asked about laboratory values using a multiple-choice list, while they were asked about manifestations using an open question. Thus, responses regarding manifestations depended more on the physicians' judgment

European Medicines Agency, EMA), and laid down in the summary of product characteristics for belimumab, to initially administer belimumab for at least 6 months before evaluation of the treatment result and before any decision about continuation of the treatment [13]. Six patients discontinued the study before this time point (see details below).

A formal tool to measure disease activity was used for 76 patients (79\%), at baseline and after the initial 6 months of belimumab therapy. Here, the physicians most frequently reported
SLEDAI/SELENA-SLEDAI scores (for 65 patients; score range from 0 (no disease activity) to 105), with a mean score of $10.6 \pm 6.09$ at baseline (min 0, max 28), followed by the ECLAM (for 19 patients; range from 0 (no disease activity) to 10 ), with a mean score of $2.9 \pm 2.03$ (min 0 , max 7). The Physician Global Assessment Scale was used for 17 patients (range from 0 (no disease activity) to 100) and the mean baseline score was reported as $71.9 \pm 13.56(\min 30$, max 88), and the Patient Global Assessment Scale (performed for eight patients; range from 0 (no 
disease activity) to 100) showed a mean score of $77.5 \pm 11.65$ ( $\min 60, \max 90)$. The BILAG assessment (British Isles Lupus Assessment Group; results provided as a range from 0 (no disease activity) to 72) was performed for five patients with a mean score of $10.2 \pm 4.66$ (min 5 , max 16) at baseline.

\section{Outcomes of Belimumab Therapy After Initial 6 Months}

The patients' overall clinical response to belimumab was assessed by their physician after 6 months of treatment. For the majority of patients, improvement between 20\% and $79 \%$ was documented (Fig. 1). An overall clinical improvement of at least $20 \%$ was observed in $78 \%$ of patients and an improvement of at least $50 \%$ in $42 \%$ of patients.

When assessing the six most common clinical and serological manifestations of SLEarthritis, increased anti-dsDNA antibody levels, low complement, fatigue, rash, and alopeciamost manifestations showed improvement after 6 months of belimumab therapy for the majority of patients, as assessed by the physicians (Fig. 2). Notably high improvement levels were reported for a large proportion of patients with arthritis and rash, while complement levels showed the least improvement overall.

Improvements were also seen in the disease activity assessments (Table 2). The mean SLEDAI/SELENA-SLEDAI score decreased from 10.6 to 5.6 during the first 6 months of therapy. Similarly, most of the other commonly used indexes, the ECLAM, the Physician Global Assessment Scale, and the Patient Global Assessment Scale all showed lower, i.e., improved scores after 6 months of belimumab therapy.

\section{Discontinuations Before End of Initial 6 Months of Belimumab Therapy}

Six patients discontinued belimumab treatment within 6 months; at the time of discontinuation they had received belimumab for an average of $64 \pm 51.85$ days (min 15, $\max 155$ days). Consequently, no results for the time point after 6 months of belimumab treatment are available for these patients and they could not be included in the analyses after 6 months presented above. The six patients had ratings of "worse" (four patients), "no improvement" (one patient) and "<20\% improvement" (one
Fig. 1 Physicians' evaluation of clinical response of their systemic lupus erythematosus patients $\quad(N=96)$ after 6 months of belimumab therapy, compared to status at start of belimumab treatment

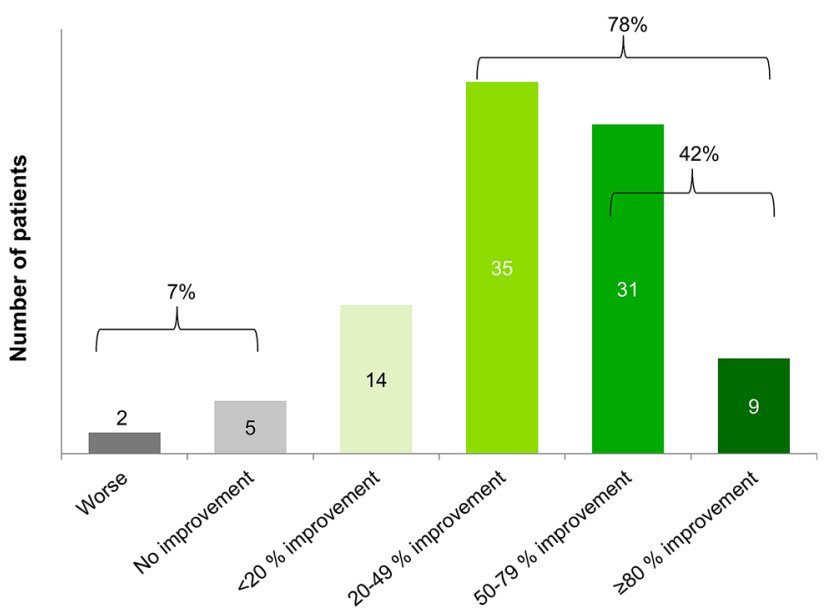




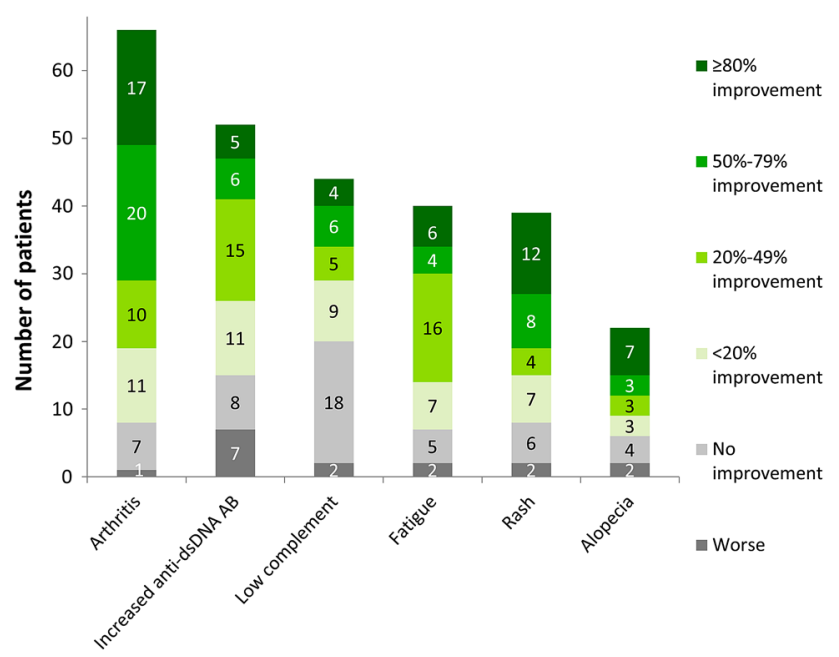

Fig. 2 Physicians' evaluation of clinical response for the most frequently reported clinical and serological manifestations of SLE in their patients: improvement rating after 6 months of belimumab therapy, compared to status at start of belimumab treatment (arthritis, $N=66$; increased anti-dsDNA antibody levels, $N=52$; low complement levels, $N=44$; fatigue, $N=40$; rash, $N=39$; alopecia, $N=22$; for all 96 patients who completed the initial 6 months of belimumab)
Table 2 SLE disease activity scores (SLEDAI/SELENA-SLEDAI, ECLAM, Physician and Patient Global Assessment Scales, BILAG) at the start of belimumab therapy and after the initial 6 months of treatment, as well as calculated changes to baseline, for all patients for whom scores were available for both time points $(N=76)$

\begin{tabular}{lcccc}
\hline Assessment tool & $\boldsymbol{N}$ & $\begin{array}{l}\text { Mean score } \mathbf{\pm} \text { SD } \\
\text { at baseline }\end{array}$ & $\begin{array}{l}\text { Mean score } \pm \text { SD after } \\
\mathbf{6} \text { months of therapy }\end{array}$ & Mean change \pm SD \\
\hline SLEDAI/SELENA-SLEDAI $^{\mathrm{a}}$ & 65 & $10.6 \pm 6.09$ & $5.6 \pm 4.07$ & $-4.9 \pm 4.94$ \\
ECLAM $^{\mathrm{b}}$ & 19 & $2.9 \pm 2.03$ & $1.8 \pm 1.70$ & $-1.0 \pm 1.40$ \\
Physician Global Assessment Scale $^{\mathrm{c}}$ & 17 & $71.9 \pm 13.56$ & $47.1 \pm 19.84$ & $-24.8 \pm 18.55$ \\
Patient Global Assessment Scale $^{\mathrm{c}}$ & 8 & $77.5 \pm 11.65$ & $27.5 \pm 17.53$ & $-50.0 \pm 25.63$ \\
BILAG $^{\mathrm{d}}$ & 5 & $10.2 \pm 4.66$ & $10.2 \pm 7.19$ & $0.0 \pm 4.53$ \\
\hline
\end{tabular}

BILAG British Isles lupus assessment group index, ECLAM European consensus lupus activity measurement index, $N$ number of patients, SELENA "Safety of Estrogens in Lupus Erythematosus National Assessment" modification of SLEDAI, SLE systemic lupus erythematosus, SLEDAI SLE disease activity index

${ }^{a}$ (SELENA-)SLEDAI scale: Final score ranges between 0 (no disease activity) and 105

${ }^{b}$ ECLAM scale: Final score is between 0 (no disease activity) and 10

c Global Assessment Scales based on the physicians' internal rating system; values were converted to a scale ranging from 0 to 100 based on ranges provided by the physicians

d BILAG range: Final score between 0 (no disease activity) and 72

patient) in the physicians' evaluation of their clinical response. In two of these patients a new induction therapy with cyclosporine or cyclophosphamide was initiated after the start of belimumab. Details of the six cases of discontinuation are presented in Table 3. 
Table 3 Details of patients who discontinued belimumab therapy before the end of the first 6 months of belimumab therapy $(N=6)$

\begin{tabular}{|c|c|c|c|c|c|}
\hline Case & $\begin{array}{l}\text { Severity of } \\
\text { SLE at } \\
\text { baseline }\end{array}$ & $\begin{array}{l}\text { Duration of } \\
\text { belimumab } \\
\text { exposure (days) }\end{array}$ & $\begin{array}{l}\text { Assessed } \\
\text { change in SLE } \\
\text { severity }^{\text {a }}\end{array}$ & Reason(s) for discontinuation & $\begin{array}{l}\text { Corticosteroid } \\
\text { dose change }\end{array}$ \\
\hline 1 & Moderate & 16 & Worse & $\begin{array}{l}\text { Disease progression and ineffective } \\
\text { medication }\end{array}$ & $\begin{array}{l}\text { No change } \\
(5 \mathrm{mg} / \text { day })\end{array}$ \\
\hline 2 & Moderate & 28 & $\begin{array}{l}<20 \% \\
\text { improved }\end{array}$ & $\begin{array}{l}\text { AE: allergic reaction (relationship } \\
\text { suspected }^{\mathrm{b}} \text { ) }\end{array}$ & $\begin{array}{l}\text { Increase ( } 9 \text { to } \\
10 \mathrm{mg} / \text { day) }\end{array}$ \\
\hline 3 & Moderate & 35 & Worse & Disease progression & $\begin{array}{l}\text { No change } \\
\qquad(5 \mathrm{mg} / \text { day })\end{array}$ \\
\hline 4 & Unknown & 68 & Worse & $\begin{array}{l}\text { Disease progression and ineffective } \\
\text { medication; AE: lupus myelopathy } \\
\text { (relationship not suspected }^{\text {b }}\end{array}$ & $\begin{array}{l}\text { Reduction }(20 \\
\text { to } \\
15 \mathrm{mg} / \text { day })\end{array}$ \\
\hline 5 & Moderate & 87 & None & $\begin{array}{l}\text { Patient request and lack of patient } \\
\text { compliance }\end{array}$ & $\begin{array}{l}\text { No change } \\
\qquad(5 \mathrm{mg} / \text { day })\end{array}$ \\
\hline 6 & Moderate & 156 & Worse & $\begin{array}{l}\text { SAE: death due to undiagnosed } \\
\text { cardiomyopathy/heart failure } \\
\text { (relationship not suspected }^{\text {b }} \text { ) }\end{array}$ & $\begin{array}{l}\text { No change } \\
(250 \mathrm{mg} / \text { day } \\
\text { IV })\end{array}$ \\
\hline
\end{tabular}

$A E$ adverse event, $S A E$ serious adverse event, $S L E$ systemic lupus erythematosus, $I V$ intravenous

a SLE improvement from start of belimumab therapy (baseline) to time of discontinuation

b Relationship to belimumab treatment, according to AE reporting form

c Change in dose of concomitant corticosteroid medication from baseline to time of discontinuation

\section{Treatment with Other SLE Medications Before and During Belimumab Treatment}

All patients in this study (including those who discontinued) had undergone treatment with at least two other SLE medications (mean number of other medications $5.5 \pm 1.96$, $\min 2$, $\max 10$ ) prior to starting belimumab therapy: for $50 \%$ of the patients, more than five other previous medications had been documented. All patients were receiving other SLE medications at the start and during the 6 months of belimumab therapy, most commonly oral corticosteroids, antimalarials, as well as the immunosuppressants mycophenolate mofetil and azathioprine. During the first 6 months with a slowly evolving belimumab effect three patients also received a new induction treatment with cyclophosphamide and one patient has been started cyclosporine. Two of those patients discontinued belimumab.

The usage of most SLE medications among the 96 patients who completed the initial 6 months of belimumab treatment remained stable (Table 4).

A total of 91 patients who completed the 6 months' course of belimumab therapy received oral corticosteroids at the start or during this therapy and were evaluated regarding their daily corticosteroid intake. A notable dose reduction of oral corticosteroids was observed for these patients (Fig. 3).

The mean dose of corticosteroids was $11.7 \mathrm{mg} /$ day 6 months before belimumab 
Table 4 Other medications for SLE that the patients in the OBSErve Germany study received in the 6 months prior to starting belimumab therapy, at the time of initiating belimumab, and during the therapy $(N=96)$

\begin{tabular}{|c|c|c|c|}
\hline SLE medication ${ }^{a}$ & $\begin{array}{l}\text { During the } 6 \text { months before } \\
\text { belimumab therapy } \\
\text { Number of patients (\%) }\end{array}$ & $\begin{array}{l}\text { At belimumab } \\
\text { initiation } \\
\text { Number of patients (\%) }\end{array}$ & $\begin{array}{l}\text { During the first } 6 \text { months } \\
\text { of belimumab therapy } \\
\text { Number of patients (\%) }\end{array}$ \\
\hline Oral corticosteroids & $85(89 \%)$ & $90(94 \%)$ & $88(92 \%)$ \\
\hline Antimalarials & $53(55 \%)$ & $57(59 \%)$ & $55(57 \%)$ \\
\hline Mycophenolate mofetil & $27(28 \%)$ & $25(26 \%)$ & $24(25 \%)$ \\
\hline Azathioprine & $17(18 \%)$ & $15(16 \%)$ & $15(16 \%)$ \\
\hline Methotrexate & $12(13 \%)$ & $11(11 \%)$ & $8(8 \%)$ \\
\hline Cyclosporine & $9(9 \%)$ & $6(6 \%)$ & $7(7 \%)$ \\
\hline NSAIDs & $6(6 \%)$ & $6(6 \%)$ & $5(5 \%)$ \\
\hline Cyclophosphamide & $7(7 \%)$ & $1(1 \%)$ & $2(2 \%)$ \\
\hline IV corticosteroids & $2(2 \%)$ & $1(1 \%)$ & $0(0 \%)$ \\
\hline Thalidomide & $1(1 \%)$ & $1(1 \%)$ & $1(1 \%)$ \\
\hline Dapsone & $1(1 \%)$ & $0(0 \%)$ & $0(0 \%)$ \\
\hline
\end{tabular}

$I V$ intravenous, $N S A I D s$ non-steroidal anti-inflammatory drugs, $S L E$ systemic lupus erythematosus

${ }^{\text {a }}$ Multiple responses possible

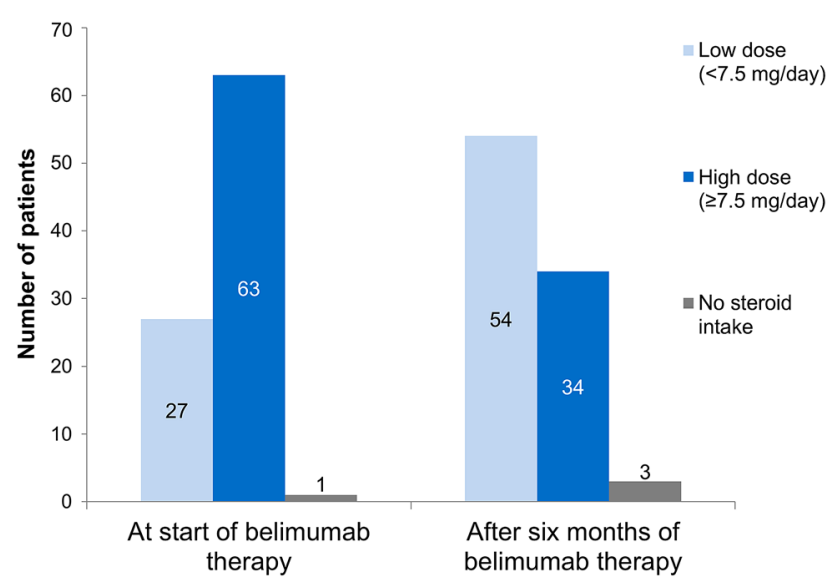

Fig. 3 Number of patients in high and low oral corticosteroid dose groups, at start of belimumab therapy, and after the initial 6 months of belimumab therapy (for

therapy, $13.7 \mathrm{mg} /$ day at belimumab start, and $7.6 \mathrm{mg} /$ day after 6 months of treatment. A similar reduction of the mean dose was also seen when analyzing only those 63 patients who were being treated with a dose of all patients who were taking oral corticosteroids and who completed the initial 6 months of belimumab treatment, $N=91)$

corticosteroids of at least $7.5 \mathrm{mg} /$ day at the start of belimumab treatment (Table 5). Of these 63 patients, 29 had switched to a low dose (less than $7.5 \mathrm{mg} /$ day) after 6 months of belimumab therapy. Those 32 patients who remained on a 
Table 5 Mean doses of oral corticosteroids 6 months before belimumab therapy, at initiation of belimumab therapy, and 6 months later

\begin{tabular}{llll}
\hline & $\begin{array}{l}\text { Total } \\
(\boldsymbol{N}=\mathbf{9 1})^{\mathbf{a}}\end{array}$ & $\begin{array}{l}\text { Patients on } \\
\text { corticosteroid } \geq 7.5 \mathbf{~ m g} / \text { day at } \\
\text { belimumab start }(\boldsymbol{N}=\mathbf{6 3})^{\mathbf{a}}\end{array}$ & $\begin{array}{l}\text { Patients on } \\
\text { corticosteroid }<7.5 \text { mg/day at } \\
\text { belimumab start }(\boldsymbol{N}=\mathbf{2 7})^{\mathbf{a}}\end{array}$ \\
\hline $\begin{array}{l}\text { Mean dose }( \pm S D) 6 \text { months } \\
\text { before belimumab start } \\
(\mathrm{mg} / \text { day })\end{array}$ & $11.7 \pm 15.00$ & $12.6 \pm 13.25$ & $10.2 \pm 18.73$ \\
$\begin{array}{l}\text { Mean dose }( \pm S D) \text { at belimumab } \\
\text { start (mg/day) }\end{array}$ & $13.7 \pm 13.75$ & $17.5 \pm 15.04$ & $5.4 \pm 1.11$ \\
$\begin{array}{l}\text { Mean dose ( } \pm \text { SD) after first } \\
\text { 6 months of belimumab } \\
\text { therapy (mg/day) }\end{array}$ & $7.6 \pm 5.99$ & $8.6 \pm 6.63$ & $5.2 \pm 3.36$ \\
\hline
\end{tabular}

Data are shown for all patients who received oral corticosteroids at one of these time points and who completed the initial 6 months of belimumab therapy $(N=91)$, as well as for all patients who initiated belimumab treatment while taking a dose $\geq 7.5 \mathrm{mg}$ /day $(N=63)$ or a low dose $(N=27)$ of oral corticosteroids

a One patient initiated corticosteroid intake after start of belimumab therapy and was therefore only included in the "Total" column, not in the subgroups by starting dose

high dose still showed a reduction in their mean dose (from $16.0 \pm 9.94$ to $12.2 \pm 7.61 \mathrm{mg} /$ day). Two patients discontinued oral corticosteroid use during belimumab treatment. Almost all of the 27 patients who were on a low corticosteroid dose (less than $7.5 \mathrm{mg} /$ day) at the start of belimumab treatment remained on the low dose, showing a slight decrease in this dose from $5.3 \pm 1.11$ to $4.8 \pm 1.34 \mathrm{mg} /$ day after 6 months of belimumab therapy. One patient discontinued oral corticosteroids during this time and one patient changed from a low to a high dose. One patient in the study started taking oral corticosteroids (at least $7.5 \mathrm{mg} /$ day) during the belimumab treatment period.

\section{Utilization of Other Medications and Healthcare Resources}

During the first 6 months of belimumab therapy, the use of non-SLE medications among the 96 patients who completed the initial 6 months of belimumab therapy remained relatively constant. The number of patients requiring SLE-related emergency room visits dropped from $9 \%$ to $3 \%$ in the first 6 months of belimumab therapy, as did the number of SLE-related hospitalizations, with $24 \%$ of patients requiring hospitalisation before start of belimumab therapy in comparison to $11 \%$ of patients during belimumab treatment (Table 6). While the number of scheduled visits to the physician's practice per patient increased during belimumab therapy due to the infusions, the number of unscheduled visits was reduced overall.

\section{DISCUSSION}

The OBSErve Germany study reflects the real-world practice of treating SLE with belimumab after its approval in Germany 2011. As a result of its purely retrospective character and the methodological approach, the 
Table 6 Unscheduled health resources utilization documented for the SLE patients in the OBSErve Germany study during the initial 6 months of belimumab therapy, in comparison to the 6 months before start of belimumab treatment $(N=96)$

\begin{tabular}{|c|c|c|c|c|}
\hline & \multicolumn{2}{|c|}{$\begin{array}{l}\text { During the } 6 \text { months before start of } \\
\text { belimumab therapy }\end{array}$} & \multicolumn{2}{|c|}{$\begin{array}{l}\text { During the initial } 6 \text { months of } \\
\text { belimumab therapy }\end{array}$} \\
\hline & Number of patients & $\%$ of patients & Number of patients & \% of patients \\
\hline \multicolumn{5}{|l|}{ SLE-related emergency room visits } \\
\hline Yes & 9 & 9 & 3 & 3 \\
\hline No & 1 & 1 & 7 & 7 \\
\hline \multicolumn{5}{|l|}{ Hospitalizations } \\
\hline$\geq 2$ & 8 & 8 & 0 & 0 \\
\hline 1 & 15 & 16 & 11 & 11 \\
\hline 0 (or date unknown) & 73 & 76 & 85 & 89 \\
\hline Hospitalization related to belimumab & 0 & 0 & 0 & 0 \\
\hline
\end{tabular}

SLE systemic lupus erythematosus

results are only descriptive for the patients included and no formal conclusions regarding the effectiveness and tolerability of belimumab can be drawn for populations other than this.

The OBSErve Germany study aimed to elucidate how belimumab is being used in routine clinical care in Germany (its initiation and discontinuation), 2 years after its marketing authorization. The patient data included in the study were sourced from 21 clinical sites with experience not only in treating SLE patients but also in prescribing belimumab. The demography of the patient population was as expected for SLE patients in Germany, including predominantly white females [27]. Most of the 102 documented patients had been living with SLE for a number of years and had moderate or severe disease. Many of them were serologically active with high levels of anti-dsDNA antibodies and below-normal levels of complement components in their blood and were suffering from several clinical SLE manifestations like arthritis, nephritis or rash, and from other co-morbid conditions such as fatigue, hypertension, and osteoporosis. Belimumab therapy was most commonly initiated because previous treatments had been regarded as ineffective. Thus, the study population included mainly patients with a long and severe SLE history not sufficiently controlled by other medications. Reflecting this, all patients had been taking a number of other SLE medications before starting belimumab therapy and also continued taking other drugs along with belimumab, most frequently oral corticosteroids. In 26 patients a manifestation of mild lupus nephritis was present together with other manifestations. Of note, belimumab is not recommended in patients with severe acute renal involvement (serum creatinine greater than $2.5 \mathrm{mg} / \mathrm{dl}$, proteinuria greater than $6 \mathrm{~g} /$ day) or acute neuropsychiatric lupus, that were not present in our cohort. Overall, the baseline characteristics showed that belimumab is being prescribed appropriately in routine care; it is indicated as an additional therapeutic measure for patients with high disease activity 
based on clinical and laboratory parameters who show autoreactive antibodies and who have not responded sufficiently to other SLE treatments [13]. Owing to the relatively large sample size (about one-third of all patients being treated with belimumab in Germany at that time) and the efforts to minimize selection bias by including all eligible patient records at each participating site, the results provide a first indication of the belimumab treatment situation in Germany between 2011 and 2013, with a documentation period from April to November 2013.

The patients' clinical outcomes after 6 months of belimumab treatment were evaluated by the participating physicians on the basis of their patients' medical records. On the one hand, the physicians provided a subjective assessment of their individual patients' SLE status improvement after belimumab treatment based on a non-validated but true-to-life scale. On the other hand this outcome assessment often was complemented by standardized disease activity scores. The physicians' judgment was favorable for the majority of patients, with $74 \%$ of patients showing an overall SLE status improvement of at least $20 \%$ and $42 \%$ of patients an improvement of at least $50 \%$. Similarly, most of the frequent clinical and serological manifestations showed improvements after 6 months, as assessed by the responsible physician. In addition, 19 of the participating 21 physicians stated that they defined a priori treatment targets allowing the measurement of the achievements against the targets and to use formal disease activity scores on a regular basis as recommended by international guidelines (e.g., EULAR) [26]. Therefore, results from such scores are available for 79 of the 102 patients in this study, possibly indicating a recently extended use of these tools, at least for the newer and more expensive treatment options. The most commonly used scores were the SLEDAI/ SELENA-SLEDAI, the ECLAM, the Patient Global Assessment Scale, and the Physician Global Assessment Scale, all of which showed lower, i.e., improved, scores after 6 months of belimumab therapy. This combination of subjective physicians' assessments and validated disease activity indexes appears to reflect a positive trend in clinical SLE management; new composite response indexes which incorporate the physicians' assessment have recently been developed [28]. The world's largest as yet conducted lupus studies, the randomized controlled BLISS trials with belimumab, used the Physician Global Assessment (PGA) as part of the combined SRI index (SLE Responder Index) [29]. The SRI, however, is not feasible for the assessment of lupus activity in daily clinical routine practice mainly because of its BILAG part. In contrast, the PGA, as a subjective and non-standardized evaluation of effectiveness, still is the basis for most treatment decisions. The fact that the subjective assessment of disease activity is consistent with the results from formal scores and that the validated tools are being used on a regular basis for the majority of patients documented in the OBSErve Germany study may indicate that the recommended approach of systematically assessing a patient's condition over time is becoming more common in clinical practice. This is a real-world finding of OBSErve Germany that complements the more controlled objectives addressed by the clinical trials.

Consistent with the clinical outcomes, a reduction in the use of oral corticosteroid medication was also observed. The association of corticosteroids with severe side effects at higher doses (at least $7.5 \mathrm{mg} /$ day; [30]) makes 
any corticosteroid reduction to at least below this threshold one of the key therapeutic targets in SLE management [7]. After the initial 6 months of belimumab therapy, the mean dose of corticosteroids taken by the patients had decreased markedly and more than half of the patients who had entered belimumab treatment on a high dose of corticosteroids were able to change to a low dose. While the randomized clinical trials with belimumab were not designed to show a steroid-sparing effect, the OBSErve Germany results contribute to the growing set of data describing such outcomes. If the potential link between belimumab treatment and corticosteroid sparing can be proven in further clinical or observational studies, it will be highly relevant for the management of SLE patients, especially for those who do not cope well with the side effects of corticosteroids and/or who could not achieve a dose within an acceptable range before. In contrast to the corticosteroid intake, the use of other concomitant medications was not reduced in this study.

Finally, the extent of utilization of healthcare resources was evaluated for the study population. As an innovative antibody therapy, belimumab treatment is more costly than other SLE medications. However, its benefits appear to impact other healthcare resources in routine clinical care. In the OBSErve Germany study, the patients required fewer SLE-related emergency room visits and fewer SLE-related hospitalizations in the 6 months of belimumab therapy than in the 6 months before. The number of scheduled visits of patients at their physician's practice increased on average, as expected during the course of belimumab, which requires administration as monthly infusions. The number of unscheduled visits, on the other hand, was reduced. Overall, it appears that belimumab-treated patients have fewer SLE-related emergencies and that their SLE activity management is improved within the first 6 months of the treatment.

OBSErve Germany was a non-interventional study. Such investigations have certain inherent strengths (e.g., the real-world perspective, potentially large patient populations, longer investigation periods) as well as limitations (e.g., no stringent evaluation of efficacy, no source data verification, possible issues with availability and comparability of diagnostic data, limited control of confounding effects) in comparison to clinical trials [31]. As discussed above, the real-world setting provided insights into how belimumab is used in routine care, when no protocol or monitoring is required. Interference with the physicians' procedures was impossible because of the retrospective data collection. The data provided by the physicians were checked for plausibility but not verified in the source documents. No control group was set up. Consequently, no direct conclusions regarding the efficacy and safety of belimumab can be drawn. Nevertheless, the results of the OBSErve Germany study do indicate good effectiveness and the low number of discontinuations due to adverse events (AEs) indicates good tolerability, in line with the clinical trials where efficacy and safety were confirmed previously.

OBSErve Germany was designed with a different focus; complementary to the pivotal studies it is important to understand the practicalities of belimumab treatment, outcomes, and discontinuations under real-world conditions to determine the clinical value of this new therapy and its potential impact on SLE management [25]. A detailed profile of those patients that respond particularly well to belimumab treatment can shape future treatment guidelines and help 
physicians identify the right treatment for a patient. SLE is a disease where many patients rely on a combination of two or three medications to manage disease activity. Therefore, the effect of belimumab on the concomitant SLE medication may be of interest in further investigations covering a longer time period. Compared to the patient population in the BLISS studies [14, 15], the patients documented in the OBSErve Germany study had a higher mean disease activity. The observations described here are highly consistent with a subgroup analysis of the BLISS patients which shows that patients with higher disease activity are the ones responding best to belimumab [16]. On the basis of these results, further SLE subgroups may be of interest for assessing the risks and benefits of belimumab treatment. For example, a large clinical trial is currently ongoing to investigate belimumab treatment outcomes specifically for adult patients with active lupus nephritis.

As a result of the retrospective and observational nature of the OBSErve study, safety data were not collected in detail. However, the data on discontinuations may provide some insight into safety aspects. Six of the 102 study patients discontinued belimumab treatment within 6 months. For three of these patients, their physicians documented "disease progression" and "ineffective medication" as the reasons for withdrawing belimumab. In these patients, treatment was discontinued after 16, 35, and 68 days, respectively, i.e., within the first 10 weeks. This is a point in time where the full clinical effect of belimumab cannot yet be expected. As s result of its mode of action and supported by the clinical study results, a minimum treatment period of 6 months is recommended in the summary of product characteristics for belimumab [13], to allow full efficacy to develop before a decision regarding further therapy is made. In the three patients described here, the constellation of their course of disease and their concomitant corticosteroid doses at least leaves some questions open, one of them being whether more patience regarding the development of the therapeutic effects could have led to different treatment results. Two of the patients discontinued because of an adverse event, one of which was an allergic reaction. This is consistent with the summary of product characteristic for belimumab, which describes that $1 \%$ of belimumab study patients are withdrawn from the treatment because of an allergic reaction [13]. For the one patient who died, no causal relationship to belimumab was suspected.

These results are consistent with the conclusion from the clinical trials that belimumab is generally well tolerated although this study did not collect safety data besides those leading to withdrawal [32]. As with other immunomodulatory drugs or antibody therapies, patient monitoring remains important, however, especially during and after the first exposures.

Overall, the effectiveness of belimumab seen in the OBSErve Germany study exceeded the expectations from the pivotal trials [14-16] with regards to the extent of clinical improvement and to its onset. Whether this is a real effect or a methodological bias due to the high hurdles to achieve the primary endpoints in clinical studies needs to be evaluated further. Very similar results have been presented for patients in Spain and in the USA (OBSErve Spain, published in an abstract [24]; OBSErve US [23]). Other, smaller-scale studies assessing the effectiveness of belimumab in a real-world setting have also confirmed belimumab as an option for patients not sufficiently controlled by conventional therapies [33-37]. 


\section{CONCLUSIONS}

The OBSErve Germany study provides the first insight into belimumab use and outcomes for SLE patients in Germany, 2 years after its market launch. The real-world results reveal that, after 6 months of belimumab treatment, disease activity as well as concomitant oral corticosteroid use was reduced in the study population of patients that mostly had moderate or severe SLE and elevated autoreactive antibody levels. The low rate of discontinuation supports the good general tolerability of belimumab. Overall and despite all limitations described, belimumab is showing promising real-world results and a broad treatment response for the patient group investigated, in line with the results from the pivotal trials. Such a new therapeutic option for a subgroup of patients with higher disease activity is particularly valuable in the context of a variable disease like SLE, where the unmet medical need still is high, the quality of life markedly reduced for the affected patients, and for which no other new therapies have been approved for decades.

\section{ACKNOWLEDGEMENTS}

OBSErve Germany was sponsored by GlaxoSmithKline, Uxbridge, UK and conducted by Kantar Health $\mathrm{GmbH}$, Munich, Germany, including study coordination by Constanze Cholmakow-Bodechtel and Claudia Kanitscheider, statistical analyses by Marian Schmidt and Hans-Jörg Schwarz, and medical writing assistance by Sarah Hemer and Daniela Tonn of Kantar Health. Medical writing assistance as well as the publication charges of this article were funded by GlaxoSmithKline, Uxbridge, UK. All authors had full access to all of the data in this study and take complete responsibility for the integrity of the data and accuracy of the data analysis. All named authors meet the International Committee of Medical Journal Editors (ICMJE) criteria for authorship for this manuscript, take responsibility for the integrity of the work as a whole, and have given final approval for the version to be published. The authors thank all further participating investigators: Prof Dr. Hans-Joachim Anders, Dr. Peer M. Aries, Dr. Gunther Aßmann, Dr. Frank Behrens, Dr. Ino K. Gao, Dr. Jörg Henes, Prof Dr. Annett Jacobi, Dr. Helge Körber, Dr. Kirsten Lüthke, Prof Dr. Peter Oelzner, Dr. Elke Riechers, PD Dr. Ekkehard Röther, Dr. Christoph Schäfer, Dr. Astrid M. Thiele, Prof Dr. Hans-Peter Tony, Prof Dr. Stefan Weiner.

Disclosures A. Schwarting received personal fees from GSK during the conduct of the study. A. Schwarting also reports grants and personal fees from GSK, outside the submitted work. C. Fiehn received personal fees from GSK during the conduct of the study. J.O. Schroeder received personal fees from GSK during the conduct of the study. J.O. Schroeder also reports personal fees from GSK, outside the submitted work. M. Schmalzing received personal fees from GSK during the conduct of the study. T. Alexander received personal fees from GSK during the conduct of the study. C. Specker reports personal fees from GSK outside the submitted work. A. Perna is an employee of GSK and holds equity ownership in the company. H. Carnarius is an employee of GSK and holds equity ownership in the company. V. Koscielny was at the time of the study and manuscript preparation a GSK employee and held equity ownership in the company. C. Cholmakow-Bodechtel is an employee of Kantar Health. 


\section{Compliance with Ethics Guidelines This} article does not contain any new studies with human or animal subjects performed by any of the authors.

Open Access This article is distributed under the terms of the Creative Commons Attribution-NonCommercial 4.0 International License (http://creativecommons.org/licenses/ by-nc/4.0/), which permits any noncommercial use, distribution, and reproduction in any medium, provided you give appropriate credit to the original author(s) and the source, provide a link to the Creative Commons license, and indicate if changes were made.

\section{REFERENCES}

1. Houssiau FA, Vasconcelos C, D'Cruz D, et al. Early response to immunosuppressive therapy predicts good renal outcome in lupus nephritis: lessons from long-term followup of patients in the Euro-Lupus Nephritis Trial. Arthritis Rheum. 2004;50(12):3934-40.

2. Ginzler EM, Dooley MA, Aranow C, et al. Mycophenolate mofetil or intravenous cyclophosphamide for lupus nephritis. N Engl J Med. 2005;353(21):2219-28.

3. Lutalo PMK, Jordan N, D'Cruz DP. Which dose of steroids and which cytotoxics for severe lupus? Presse Med. 2014;(6 Pt 2):e157-65.

4. Nørgaard JC, Stengaard-Pedersen K, Nørgaard M, de Thurah A. Antimalarials in the treatment of systemic lupus erythematosus: a registry-based cohort study in Denmark. Lupus. 2015;24(3):299-306.

5. Petri M, Bechtel B, Dennis G, et al. Burden of corticosteroid use in patients with systemic lupus erythematosus: results from a Delphi panel. Lupus. 2014;23(10):1006-13.

6. Ruiz-Arruza I, Ugarte A, Cabezas-Rodriguez I, Medina JA, Moran MA, Ruiz-Irastorza G. Glucocorticoids and irreversible damage in patients with systemic lupus erythematosus. Rheumatol (Oxford). 2014;53(8):1470-6.

7. Van Vollenhoven RF, Mosca M, Bertsias G, et al. Treat-to-target in systemic lupus erythematosus: recommendations from an international task force. Ann Rheum Dis. 2014;73(6):958-67.

8. Brugos B, Sebestyen L, Tarr T, Vincze Z. Use of cyclophosphamide and other immunosuppressive drugs in the treatment of patients with lupus nephritis. Pharm. 2014;69(6):442-4.

9. Chan TM. Treatment of severe lupus nephritis: the new horizon. Nat Rev Nephrol. 2015;11(1):46-61.

10. Leone A, Sciascia S, Kamal A, Khamashta M. Biologicals for the treatment of systemic lupus erythematosus: current status and emerging therapies. Expert Rev Clin Immunol. 2015;11(1):109-16.

11. Kamal A, Khamashta M. The efficacy of novel B cell biologics as the future of SLE treatment: a review. Autoimmun Rev. 2014;13(11):1094-101.

12. Selmi C, Generali E, Massarotti M, Bianchi G, Sciré CA. New treatments for inflammatory rheumatic disease. Immunol Res. 2014;60(2-3):277-88.

13. EMA. Benlysta-European public assessment report (EPAR) Product Information-EMEA/H/C/002015IB/0030/G. 2014.. http://www.ema.europa.eu/docs/en GB/document_library/EPAR_-_Product_Information/ human/002015/WC500110150.pdf. Accessed 15 Dec 2016.

14. Furie R, Petri M, Zamani $\mathrm{O}$, et al. A phase III, randomized, placebo-controlled study of belimumab, a monoclonal antibody that inhibits B lymphocyte stimulator, in patients with systemic lupus erythematosus. Arthritis Rheum. 2011;63(12):3918-30.

15. Navarra SV, Guzmán RM, Gallacher AE, et al. Efficacy and safety of belimumab in patients with active systemic lupus erythematosus: a randomised, placebo-controlled, phase 3 trial. Lancet. 2011;377(9767):721-31.

16. Van Vollenhoven RF, Petri MA, Cervera R, et al. Belimumab in the treatment of systemic lupus erythematosus: high disease activity predictors of response. Ann Rheum Dis. 2012;71(8):1343-9.

17. Parodis I, Axelsson M, Gunnarsson I. Belimumab for systemic lupus erythematosus: a practice-based view. Lupus. 2013;22(4):372-80.

18. Askanase AD, Yazdany J, Molta CT. Post-marketing experiences with belimumab in the treatment of SLE patients. Rheum Dis Clin North Am. 2014;40(3):507-17.

19. Lutalo PMK, D'Cruz DP. Update on belimumab for the management of systemic lupus erythematosus. Expert Opin Biol Ther. 2014;14(11):1701-8. 
20. Baker KP, Edwards BM, Main SH, et al. Generation and characterization of LymphoStat-B, a human monoclonal antibody that antagonizes the bioactivities of B lymphocyte stimulator. Arthritis Rheum. 2003;48(11):3253-65.

21. Cancro MP, D'Cruz DP, Khamashta MA. The role of B lymphocyte stimulator (BLyS) in systemic lupus erythematosus. $\mathrm{J} \quad$ Clin Investig. 2009;119(5):1066-73.

22. Stohl W, Hiepe F, Latinis KM, et al. Belimumab reduces autoantibodies, normalizes low complement, and reduces select B-cell populations in patients with systemic lupus erythematosus. Arthritis Rheum. 2012;64(7):2328-37.

23. Collins CE, Dall'Era M, Kan H, et al. Response to belimumab among patients with systemic lupus erythematosus in clinical practice settings: 24-month results from the OBSErve study in the USA. Lupus Sci Med. 2016;3:e000118. doi:10.1136/ lupus-2015-000118.

24. Cortes J, Andreu JL, Calvo-Alen J, et al. Evolution of patients with systemic lupus erythematous treated with belimumab in clinical practice settings. Arthritis Rheumatol. 2014;66(Suppl 11):S291.

25. Mosca M, van Vollenhoven R. New drugs in systemic lupus erythematosus: when to start and when to stop. Clin Exp Rheumatol. 2013;31(4 Suppl 78):S82-5.

26. Bertsias G, Ioannidis JPA, Boletis J, et al. EULAR recommendations for the management of systemic lupus erythematosus. Report of a Task Force of the EULAR Standing Committee for International Clinical Studies Including Therapeutics. Ann Rheum Dis. 2008;67(2):195-205.

27. Cervera R, Doria A, Amoura Z, et al. Patterns of systemic lupus erythematosus expression in Europe. Autoimmun Rev. 2014;13(6):621-9.

28. Rao V, Gordon C. Advances in the assessment of lupus disease activity and damage. Curr Opin Rheumatol. 2014;26(5):510-9.
29. Furie RA, Petri MA, Wallace DJ, et al. Novel evidence-based systemic lupus erythematosus responder index. Arthritis Rheum. 2009;61(9):1143-51.

30. Ruiz-Irastorza G, Danza A, Khamashta M. Glucocorticoid use and abuse in SLE. Rheumatol (Oxford). 2012;51(7):1145-53.

31. Silverman SL. From randomized controlled trials to observational studies. Am J Med. 2009;122(2):114-20.

32. Wallace DJ, Navarra S, Petri MA, et al. Safety profile of belimumab: pooled data from placebo-controlled phase 2 and 3 studies in patients with systemic lupus erythematosus. Lupus. 2013;22(2):144-54.

33. Askanase AD. Clinical response to belimumab in academic clincal practices. Ann Rheum Dis. 2014;73(Suppl 2):521-2.

34. Brito Zeron P, Caminal-Montero L, Chamorro A, et al. Blocking the human B lymphocyte stimulator molecule (BLyS) using a monoclonal antibody (belimumab) in Systemic lupus erythematosus: first results in real-life spanish patients with refractory disease (biogeas-Semi Registry). Ann Rheum Dis. 2014;73(Suppl 2):985.

35. Ke X, Patel J, Kan H, Eisenberg DF, Oglesby A. Characteristics and medication use patterns among belimumab users in a commercially insured population with systemic lupus erythematosus. Arthritis Rheum. 2013;65(Suppl 10):1070.

36. Iaccarino $\mathrm{L}$, Bettio $\mathrm{S}$, Ghirardello $\mathrm{A}$, et al. Preliminary data on the efficacy of belimumab in the "real-Life": a single centre experience. Proceedings of the 9th international congress on autoimmunity; 2014 26-30 March; Nice, France.

37. Majdan M, Kucharz EJ, Jeka S, Sierakowski S, Leszczynski P, Tlustochowicz W, et al. Early clinical experiences with belimumab in Polish patients with systemic lupus erythematosus. Ann Rheum Dis. 2014;73(Suppl 2):970. 